Article

\title{
Isoindigo-Based Small Molecules with Varied Donor Components for Solution-Processable Organic Field Effect Transistor Devices
}

\author{
Hemlata Patil ${ }^{1}$, Jingjing Chang ${ }^{2}$, Akhil Gupta ${ }^{1,3, *}$, Ante Bilic ${ }^{4}$, Jishan Wu ${ }^{2}$, Prashant Sonar ${ }^{5, *}$ \\ and Sheshanath V. Bhosale ${ }^{1, *}$
}

1 School of Applied Sciences, RMIT University, GPO Box 2476, Melbourne Victoria 3001, Australia; E-Mail: hemlatap2@gmail.com (H.P.)

2 Institute of Materials Research and Engineering (IMRE), A*STAR (Agency for Science, Technology and Research), 3, Research Link, Singapore 117602, Singapore;

E-Mails: chjj1234@gmail.com (J.C.); chmwuj@nus.edu.sg (J.W.)

3 Medicinal Chemistry, Monash Institute of Pharmaceutical Sciences, Monash University, Parkville Victoria 3052, Australia

4 CSIRO Manufacturing, Virtual Nanoscience Lab, Parkville Victoria 3052, Australia; E-Mail: ante.bilic@csiro.au

5 School of Chemistry, Physics and Mechanical Engineering, Queensland University of Technology, GPO Box 2434, Brisbane QLD 4001, Australia

* Authors to whom correspondence should be addressed; E-Mails: akhil.gupta@monash.edu (A.G.); sonar.prashant@qut.edu.au (P.S.); sheshanath.bhosale@rmit.edu.au (S.V.B.);

Tel./Fax: +61-3-9925-2680 (S.V.B.).

Academic Editor: Derek J. McPhee

Received: 3 August 2015 / Accepted: 15 September 2015 / Published: 18 September 2015

\begin{abstract}
Two solution-processable small organic molecules, (E)-6,6'-bis(4(diphenylamino)phenyl)-1,1'-bis(2-ethylhexyl)-(3,3'-biindolinylidene)-2,2'-dione (coded as S10) and (E)-6,6'-di(9H-carbazol-9-yl)-1,1'-bis(2-ethylhexyl)-(3,3'-biindolinylidene)-2,2'dione (coded as S11) were successfully designed, synthesized and fully characterized. S10 and S11 are based on a donor-acceptor-donor structural motif and contain a common electron accepting moiety, isoindigo, along with different electron donating functionalities, triphenylamine and carbazole, respectively. Ultraviolet-visible absorption spectra revealed that the use of triphenylamine donor functionality resulted in an enhanced intramolecular charge transfer transition and reduction of optical band gap, when compared with its carbazole
\end{abstract}


analogue. Both of these materials were designed to be donor semiconducting components, exerted excellent solubility in common organic solvents, showed excellent thermal stability, and their promising optoelectronic properties encouraged us to scrutinize charge-carrier mobilities using solution-processable organic field effect transistors. Hole mobilities of the order of $2.2 \times 10^{-4} \mathrm{~cm}^{2} /$ Vs and $7.8 \times 10^{-3} \mathrm{~cm}^{2} / \mathrm{Vs}$ were measured using S10 and $\mathbf{S 1 1}$ as active materials, respectively.

Keywords: isoindigo; solution-processable; organic field effect transistors; donor-acceptor-donor; carbazole; triphenylamine

\section{Introduction}

Over the past two decades, the design and development of new organic semiconductors has been a subject of increasing research interest since these materials are widely used as active components for electronic devices such as light emitting diodes [1], field effect transistors [2], photodiodes [3], and photovoltaic cells [4-8]. Such vast and industrial applications place high demands on both the electronic and chemical properties of materials, including chemical and thermal stability, broad absorption profile, appropriately tuned energy levels, solution-processability and charge carriers' mobility. The fulfilment of such properties constitutes a challenge for synthetic organic chemists who generally use a "structural strategy" in which blocks of distinctive electronic properties are assembled together in one chromophore through appropriate chemical coupling reactions [9].

Prior to their use as active materials for organic electronic devices, semiconductor materials were also used for optical data storage or optical switching [10,11]. Such materials/structures are typically based on a "push-pull" modular design that combines electron-rich (donor) and electron-deficient (acceptor) units connected via a $\pi$-conjugated linker $[8,12]$. Such designs have turned out to be highly successful in view of generating new semiconducting materials, controlling highest occupied molecular orbital (HOMO) and lowest unoccupied molecular orbital (LUMO) energy levels of target chromophores and tuning optoelectronic and redox properties. This approach has also been successful in tuning the solubility of target materials which is an essential requirement for the fabrication of organic electronic devices. These push-pull or donor-acceptor (D-A) modules allow an intramolecular charge transfer (ICT) transition that is beneficial for broadening absorption spectrum and narrowing the optical band gap. Most studied designs with various $\mathrm{D}-\mathrm{A}$ combinations include, but are not limited to, D-A, D-A-D, A-D-A and A-D-A-D-A. Because of the vast majority of structural blocks and advances in organic chemistry, it is not surprising that there is tremendous interest in the development of such designs by varying $\mathrm{D}-\mathrm{A}$ combinations with the goals of achieving panchromatic absorbance, appropriate energy levels and solution processability. Exploration of such designs is possible with polymeric entities as well as small organic molecules. However, small molecular semiconductors can offer advantages over polymeric counterparts in terms of ease of synthesis, introduction of structural variation, purification, less batch to batch variations and less end-group contamination. It has been realized that in order to take full advantage of the properties of small organic molecules, engineering of chemical structure as well as 
incorporation of appropriate D and/or A functionalities are highly desirable $[13,14]$. We are decidedly interested in exploring small organic molecules for their potential applications in organic electronics.

In our efforts to design and develop versatile and novel materials for organic electronic applications [15-18], we are interested in exploring the D-A-D module in particular. We [19] and others [20-23] have demonstrated that such modules facilitate the favorable $\pi-\pi$ interactions in the film, leading to an enhanced charge transport between adjacent molecules. Few examples of acceptor functionalities within a D-A-D structural motif include diketopyrrolopyrrole (DPP), 2-pyran4-ylidenemalononitrile, thiazolothiazole, naphthalene diimide (NDI) and isoindigo [20-27]. The use of isoindigo has been widely reported to develop polymeric entities for organic electronics [28,29], however, progress on small molecular semiconductors is finitely reported [8]. A material development program based on isoindigo functionality has trailed behind other emerging accepting functionalities.

In this study, we report the design, synthesis and characterisation of the optoelectronic properties of two new materials-(E)-6,6'-bis(4-(diphenylamino)phenyl)-1,1'-bis(2-ethylhexyl)-(3,3'-biindolinylidene)2,2'-dione (S10) and (E)-6,6'-di(9H-carbazol-9-yl)-1,1'-bis(2-ethylhexyl)-(3,3'-biindolinylidene)-2,2'dione (S11) — which are based on isoindigo functionality and are represented in Figure 1. The chemical structures of both the materials, $\mathbf{S 1 0}$ and $\mathbf{S 1 1}$, were confirmed by ${ }^{1} \mathrm{H}$ - and ${ }^{13} \mathrm{C}-\mathrm{NMR}$ spectroscopies and high resolution mass spectrometry. The target materials prepared in this work were found to be highly soluble in a variety of common organic solvents, such as dichloromethane, chloroform, chlorobenzene and toluene, which is a feature that is essential for the fabrication of solution-processed organic semiconductor devices. For instance, the solubility of $\mathbf{S 1 1}$ was found to be as high as $15 \mathrm{mg} / \mathrm{mL}$ of chloroform whereas S10 exerted better solubility at $22 \mathrm{mg} / \mathrm{mL}$ of chloroform, thus indicating that triphenylamine functionality can enhance the solubility profile of a target chromophore when compared with an analogue. We have fabricated the solution-processable organic field effect transistors (OFETs) using S10 and S11. Hole mobilities of the order of $2.2 \times 10^{-4}$ and $7.8 \times 10^{-3} \mathrm{~cm}^{2} / \mathrm{Vs}$ were measured using S10 and S11, respectively. This study builds upon our search for the versatile and efficient organic materials by exploring D-A-D module and is a comparative study of the effect of different donors (triphenylamine (S10) and carbazole (S11)) whilst keeping the acceptor part (isoindigo) constant.

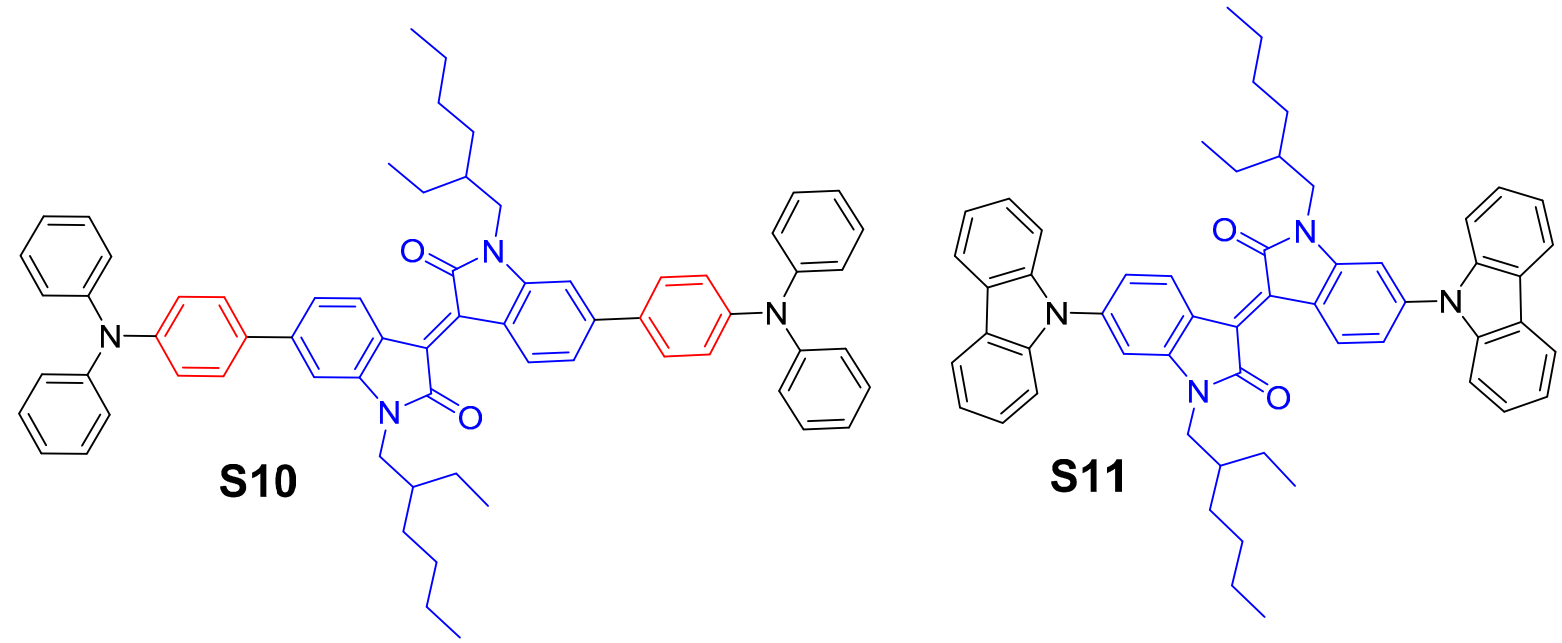

Figure 1. Molecular structures of the new organic materials investigated in this work. 


\section{Results and Discussion}

\subsection{Design Strategy, Synthesis and Characterisation}

Both materials, S10 and S11, were developed based on the D-A-D module, and the central acceptor moiety was directly linked to the donor functionalities to create a highly conjugated backbone. The development of such structures involves the use of two identical donor units placed on either side of the central core, thus resulting in a symmetrical chromophore. In S10, triphenylamine (TPA) group was selected for its believed ability to act as an energy antenna [30], which may be responsible for an overall bathochromic absorption when compared with $\mathbf{S 1 1}$ for which carbazole functionality was used. Both S10 and S11 were synthesized as per reaction Scheme 1 (For their spectra, please see Supplementary Figures S1-S8 in Supplementary Materials). The use of isoindigo functionality for enhancing the solubility of target materials is paramount as it allows an incorporation of lypophilic chains on its core nitrogen atoms. These alkyl chains facilitate the deposition of target materials as films or layers on appropriate substrates by relatively simple fabricating techniques.

\subsection{Optoelectronic Properties}

The optical properties of S10 and S11 were investigated by measuring their ultraviolet-visible (UV-Vis) absorption spectra in chloroform solution and in pristine as-casted films (Figure 2). The longest wavelength absorption maximum $\left(\lambda_{\max }\right)$ exhibited by $\mathbf{S 1 0}$ was at $572 \mathrm{~nm}$ with an absorption onset at $710 \mathrm{~nm}$. S11 exhibited its $\lambda_{\max }$ at $560 \mathrm{~nm}$ with an onset at $670 \mathrm{~nm}$. Both the absorption maximum and extinction coefficient increased with the use of TPA donor unit. This is in agreement with the design principle that the use of TPA donor unit can indeed act as an energy antenna that is helpful for stronger bathochromic shift when compared with other donor functionalities, such as carbazole. This is consistent with the idea that greater ICT can be achieved with increasing donating strength. Absorption spectra of $\mathbf{S 1 0}$ and $\mathbf{S 1 1}$ in pristine films were also measured by spin-casting their films from chloroform solutions (equimolar solutions of $\mathbf{S 1 0}$ and $\mathbf{S 1 1}$ spun at $2500 \mathrm{rpm}$ for $1 \mathrm{~min}$ ). Thin film absorption spectra exhibited bathochromic shifts when compared with solution spectra, a finding that is consistent with literature reported materials $[15,16]$. The use of TPA donor unit in S10 provided an augmentation of $\lambda_{\max }$ by $20 \mathrm{~nm}$ when compared to the carbazole unit in S11. Evidently, the use of such a strong donor can be helpful (1) to enhance the absorption profile of a given chromophore that can lead to greater light harvesting on its surface during the process of photo-excitation and (2) to intensify the $\pi$-conjugation within the molecular backbone.

We performed density functional theory (DFT) calculations on both the materials using the Gaussian 09 suite of programs [31] and B3LYP/6-311+G(d,p)//B3LYP/6-31G(d) level of theory. DFT investigation indicated that HOMO to LUMO excitation moves the orbital density distribution from the donor functionalities to the isoindigo core unit. The HOMO densities of S10 and S11 have spread across the whole molecular backbone with major dwelling over the donor functionalities. The LUMO orbital densities were delocalized over the central part of the molecule and received a sizable contribution from the isoindigo core unit (see Figure 3). This type of density distribution may be advantageous for ICT transition between donor and acceptor components. It was further observed that the use of a TPA unit as an energy antenna in $\mathbf{S 1 0}$ improves the electron-donating ability and the theoretical optical band-gap 
was reduced as a result of using TPA (2.26 eV (S10)) against carbazole functionality (2.39 eV (S11)). Experimentally, the HOMO energies of $\mathbf{S 1 0}$ and $\mathbf{S 1 1}$ were estimated using photoelectron spectroscopy in air (PESA) and the LUMO energies were calculated by adding the band gap determined by the onset of thin film UV-Vis absorption to the HOMO values.

These PESA measurements were performed on thin solid as-casted films (same films that were used to measure the absorption spectra) to measure work functions corresponding to their HOMO levels. The HOMO energy level of $\mathbf{S 1 0}$ was reduced by $0.07 \mathrm{eV}$ in comparison to the HOMO level of $\mathbf{S 1 1}$. The band gap was reduced by $0.11 \mathrm{eV}$ with the use of a TPA donor in S10. These experimental findings (1) followed the theoretical calculations trend which indicated that with the use of a strong donor, of which TPA is an example, band gap reduces and (2) that both the target chromophores are electron donating semiconducting components (see energy level diagram, Figure 4).
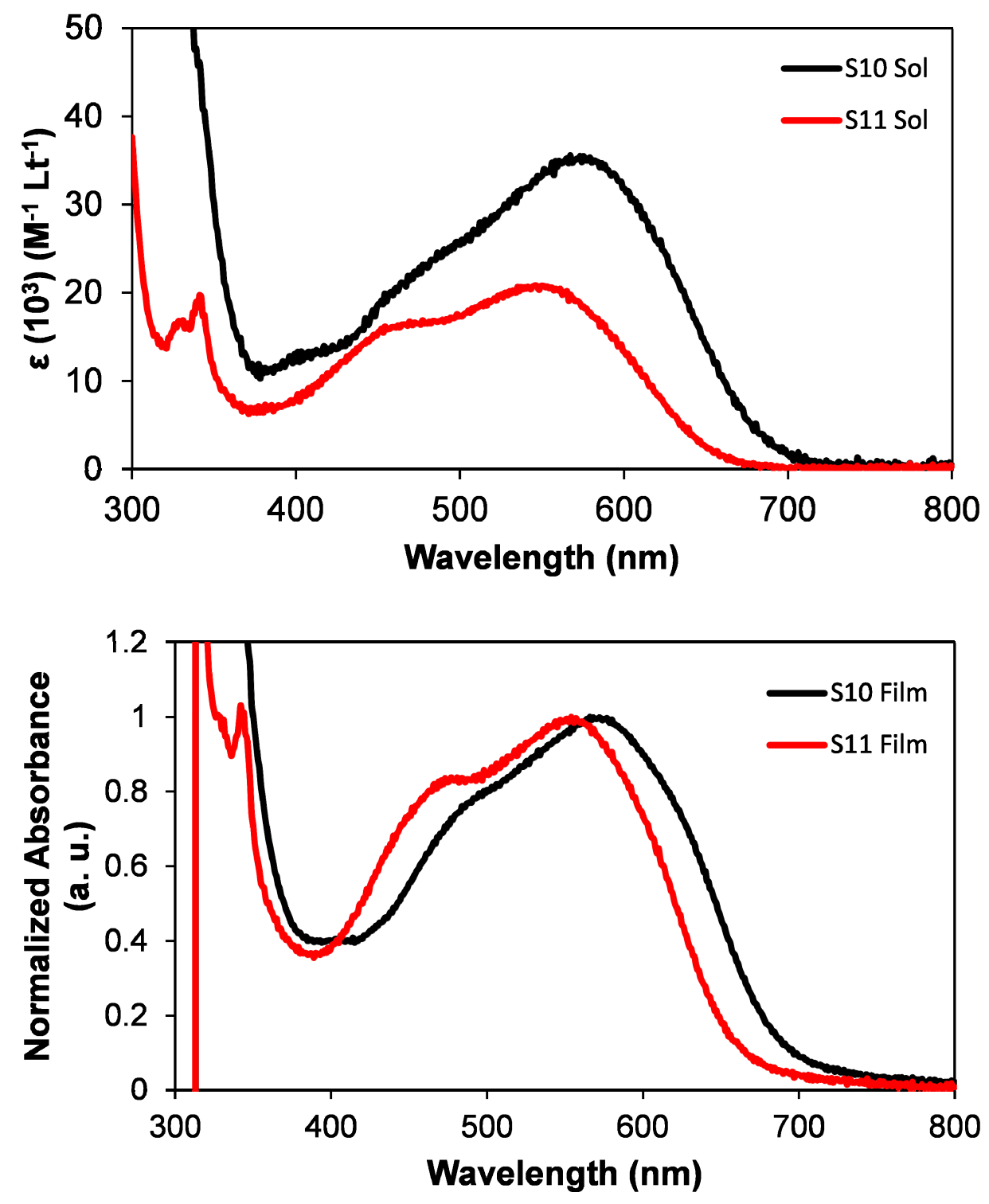

Figure 2. Molar absorptivity of newly synthesized materials S10 and S11 in chloroform solutions (upper) and normalized UV-Vis absorption spectra of S10 and S11 in thin solid films (lower), spin-cast from their chloroform solutions (equimolar solutions of S10 and S11 spun at $2500 \mathrm{rpm}$ for $1 \mathrm{~min})$. 


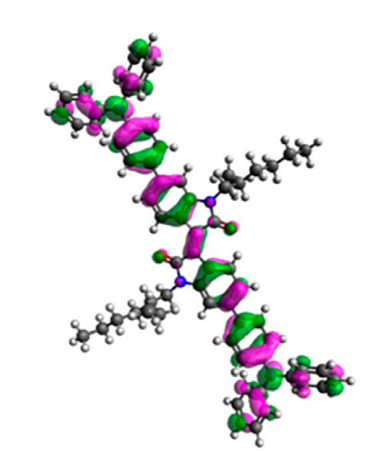

HOMO: $-5.18 \mathrm{eV}$

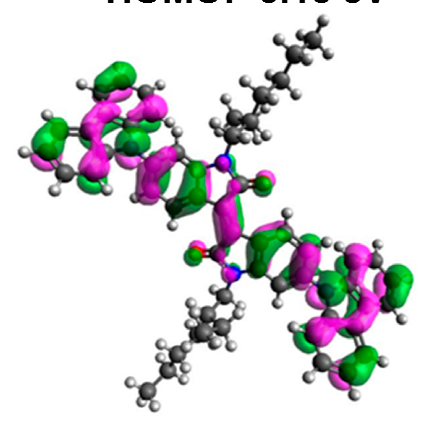

HOMO: $-5.59 \mathrm{eV}$

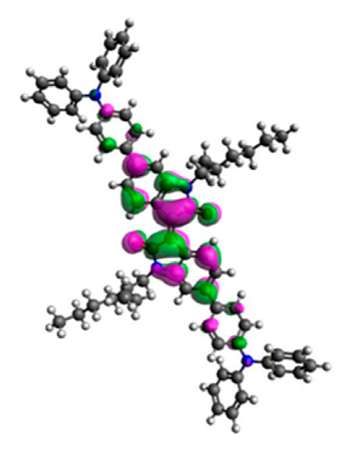

LUMO: $-2.93 \mathrm{eV}$

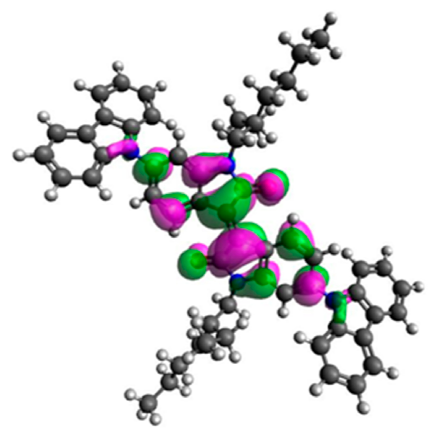

LUMO: $-3.20 \mathrm{eV}$

Figure 3. Orbital density distribution for the HOMOs and LUMOs of S10 (upper) and S11 (lower). DFT calculations on both the materials were performed using the Gaussian 09 software suite and the B3LYP/6-311+G(d,p)//B3LYP/6-31G(d) level of theory.

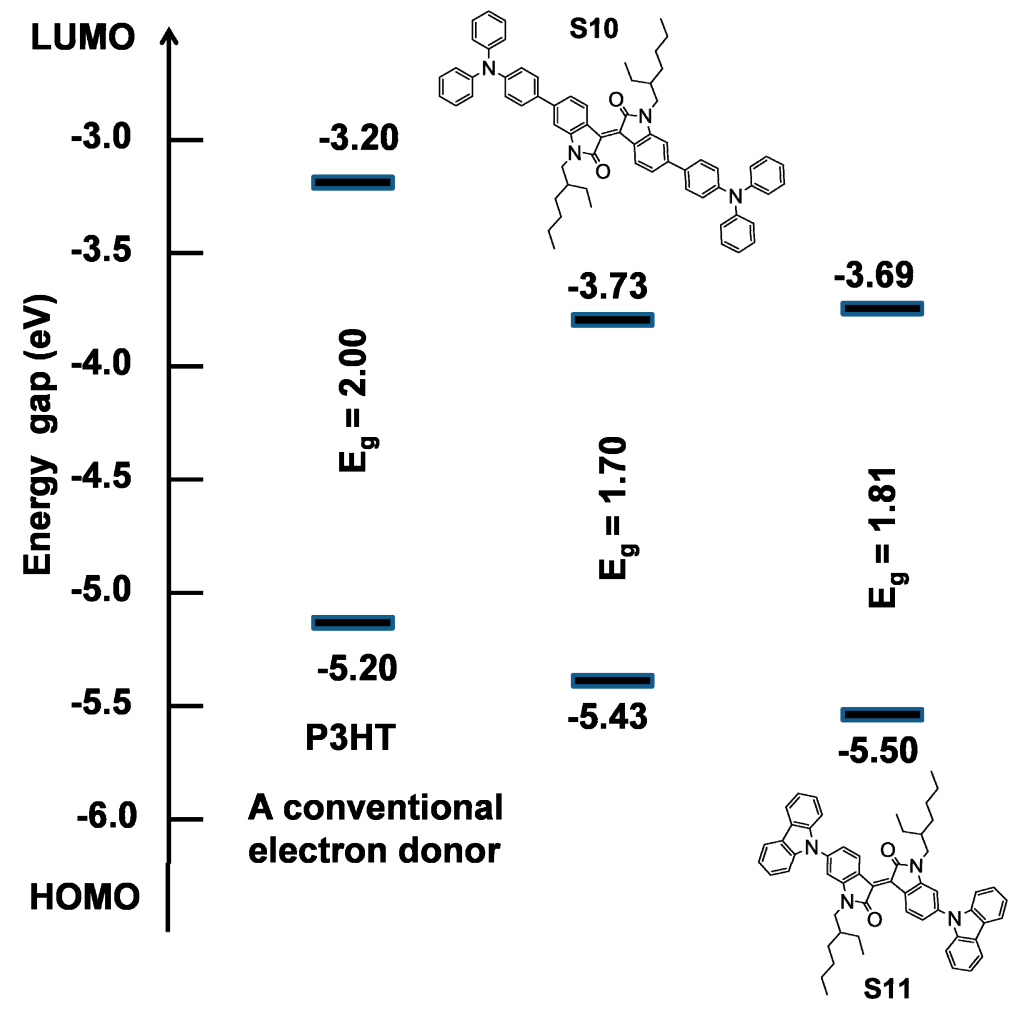

Figure 4. Energy level diagram depicting HOMOs LUMOs of S10 and S11, where HOMO levels were measured using PESA on thin solid films and LUMO levels were calculated from the optical band gaps and HOMO levels $\left(\mathrm{E}_{\mathrm{LUMO}}=\mathrm{E}_{\text {bandgap }}+\mathrm{E}_{\mathrm{HOMO}}\right)$. 
Apart from PESA measurement, we also conducted the cyclic-voltammetry (CV) experiments in order to observe the solution behavior of these materials, and to look out for reversible oxidation potentials (one or multiple) which might suggest the suitability of these materials to be used as donor semiconducting components. We conducted the CV experiments on a glassy carbon electrode. The cyclic voltammograms are included in Figure 5. Both the compounds exhibit a chemically reversible first cathodic process, thus indicating that the species formed by acceptance of an electron is stable on the voltammetry timescale. Direct connection of TPA donor to the diimide core of $\mathbf{S 1 0}$ pushes the first reduction process to a more cathodic potential relative to $\mathbf{S 1 1}$, resulting in a higher LUMO of $-3.47 \mathrm{eV}$ for $\mathbf{S 1 0}$ (calculated by the onset potential method relative to ferrocene) compared to $\mathbf{S 1 1}$, which has the LUMO energy of $-3.55 \mathrm{eV}$. This suggests that the direct bond of tertiary nitrogen atom to the central $\pi$-system in $\mathbf{S 1 1}$ reduces the electron density of electro-active centre of S11 compared to S10. Reversible oxidation potential in S10 indicates the strong donating capacity of TPA donor, an observation that verifies the design principle, absorption behavior as well as literature reports [15].

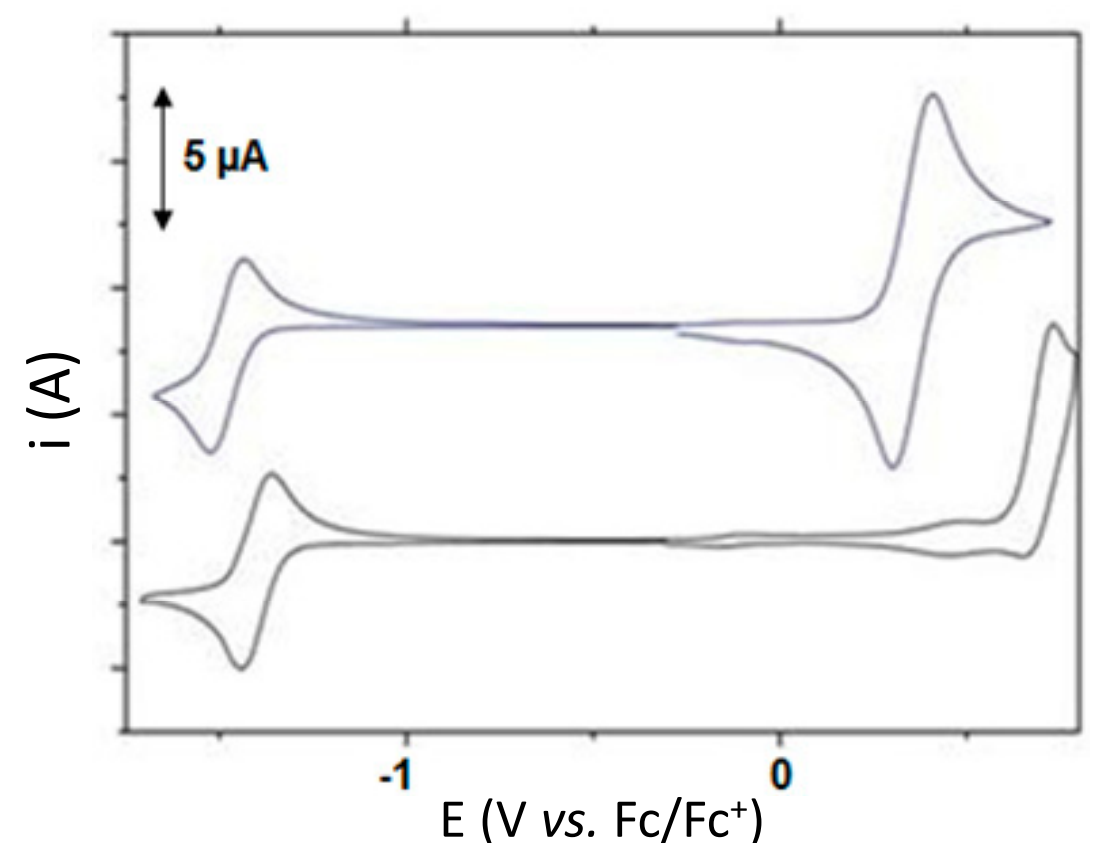

Figure 5. Cyclic-voltammograms of S10 (upper) and S11 (lower), run in freshly distilled dichloromethane at a sweep rate of $50 \mathrm{mV} \cdot \mathrm{s}^{-1}$, showing reversible reduction potential waves (both S10 and S11) and reversible oxidation potential wave (S10).

It was further realized that despite the presence of intriguing and advantageous optoelectronic properties, organic semiconducting materials must possess thermal stability so that they can sustain rigid device fabricating conditions, such as device annealing at a higher temperature. In line with this requirement, we conducted thermogravimetric and differential scanning calorimetry analyses. Thermogravimetric analysis (TGA) curves of S10 and S11 were run at a heating rate of $10{ }^{\circ} \mathrm{C} \mathrm{min}^{-1}$ under the protection of nitrogen. TGA indicated that both $\mathbf{S 1 0}$ and $\mathbf{S 1 1}$ are thermally stable up to $350{ }^{\circ} \mathrm{C}$ (Figure 6), a finding that supports high temperature annealing of as-casted organic electronic devices and corroborates differential scanning calorimetry (DSC) analysis (Supplementary Figure S9). 

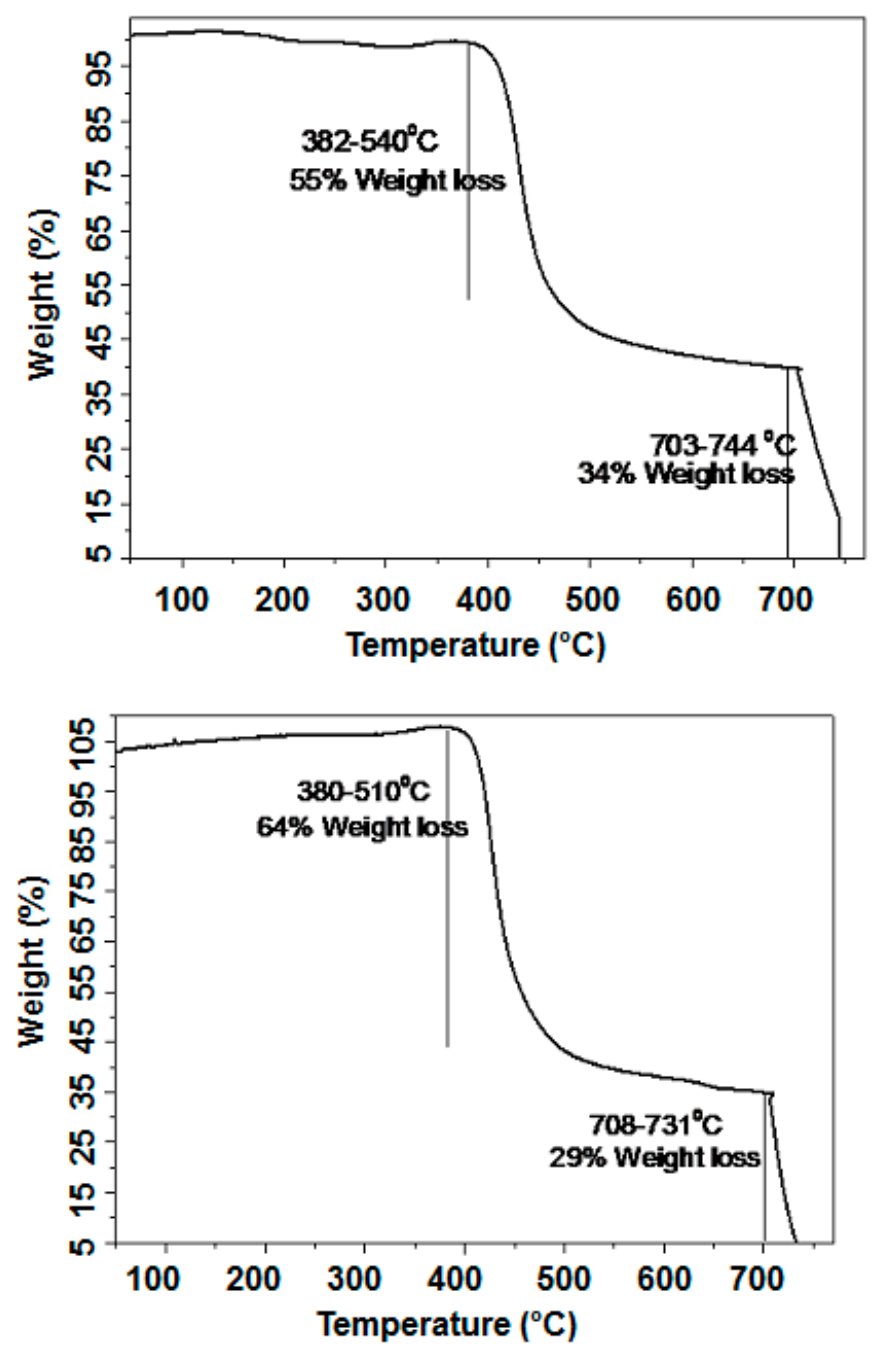

Figure 6. TGA traces of $\mathbf{S 1 0}$ (upper) and $\mathbf{S 1 1}$ (lower) under nitrogen atmosphere. Heating rate: $10{ }^{\circ} \mathrm{C} / \mathrm{min}$ from room temperature to $800{ }^{\circ} \mathrm{C}$.

The electrical properties of $\mathbf{S 1 0}$ and $\mathbf{S 1 1}$ as an active channel semiconductor in OFET devices were characterized using a bottom-gate, top-contact geometry. Heavily $n$-doped conductive silicon wafer with a layer of $\sim 200 \mathrm{~nm} \mathrm{SiO} 2$ on the surface was used as the substrate. The $\mathrm{SiO}_{2}$ functions as the gate insulator and the doped Si as the gate. The active $\mathbf{S 1 0}$ and $\mathbf{S 1 1}$ thin films ( $\sim 40 \mathrm{~nm})$ were spin coated on top of the HMDS modified $\mathrm{SiO}_{2}$ surface using $\mathbf{S 1 0}$ and $\mathbf{S 1 1} \mathrm{CHCl}_{3}$ solution $(0.5 \mathrm{wt} \%)$. On top of $\mathbf{S 1 0}$ and S11 thin films, gold was deposited as source and drain electrode via shadow mask. The OFET schematic of the complete device fabrication is shown in Figure 7a. The small molecule thin films were selectively annealed at $100{ }^{\circ} \mathrm{C}$ and $120{ }^{\circ} \mathrm{C}$ for $10 \mathrm{~min}$ on a hot plate in nitrogen atmosphere. OFET devices exhibit typical p-type electrical characteristics. The hole mobility was calculated from the saturation regime of transfer curve. The S10 and $\mathbf{S 1 1}$ thin film annealed at $120{ }^{\circ} \mathrm{C}$ exhibited hole mobility of $2.2 \times 10^{-4}$ and $7.8 \times 10^{-3} \mathrm{~cm}^{2} / \mathrm{Vs}$, respectively (Table 1). The transfer and output characteristics of $120{ }^{\circ} \mathrm{C}$ annealed S10 and S11 based OFET devices are shown in Figure 7b-e, respectively. The on/off ratios for all of the devices were calculated around $10^{4}$ to $10^{5}$ whereas the threshold voltage was observed in the range of -21 to $-16 \mathrm{~V}$ with respect to annealing. It should be noted that the interface between semiconductor and electrodes as well as the dielectric and semiconductor are critical for charge carrier injection and transport properties. Among them, the 
dielectric interface is much more crucial, and the charge carrier traps caused by silanol groups on dielectric surface could affect the electron transport more significantly. The radical anions formed are unstable towards water and oxygen due to their low LUMO energy levels (around $-3.7 \mathrm{eV}$ ), and can be easily trapped at the interface. Therefore, in our studies, only hole transport behavior was observed for these two compounds. To our knowledge, there are very few reports which explore isoindigo-based small molecules and their applications in OFET devices. The obtained hole mobility values for S10 and S11 are in good agreement with some of the previously reported small molecules based on isoindigo core [32].

(b)

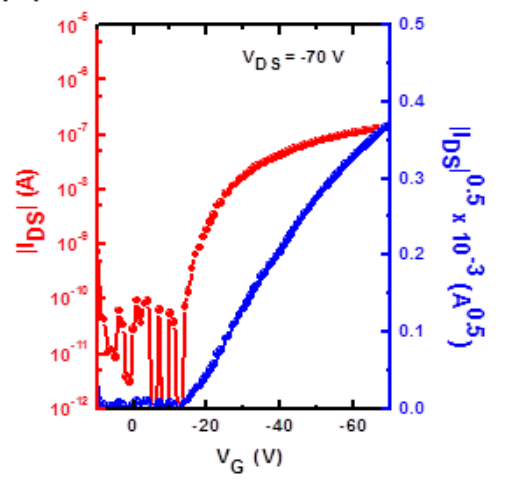

(d)

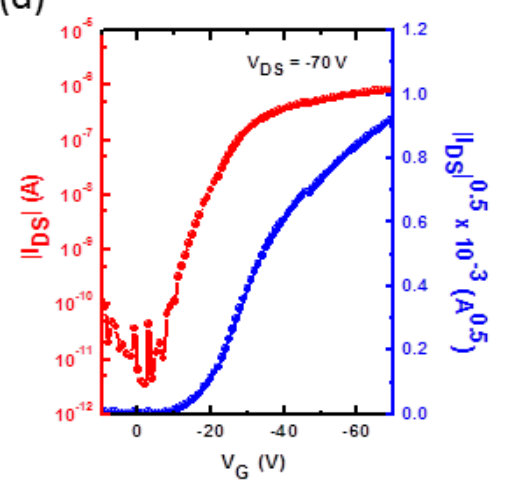

(c)

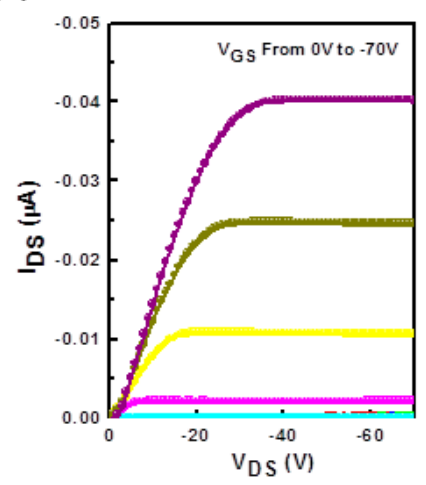

(e)

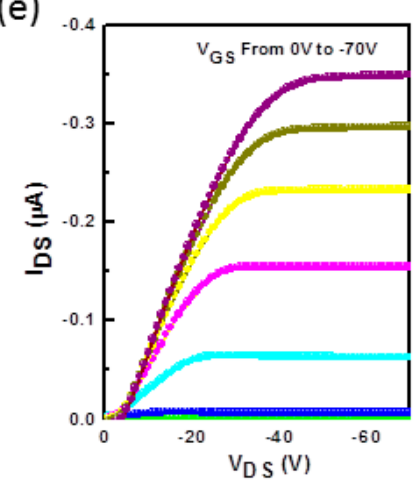

Figure 7. (a) Organic field effect transistor (OFETs) device geometry. Output and transfer characteristics of S10 (b,c) and S11 (d,e) based p-channel OFET annealed at $120{ }^{\circ} \mathrm{C}$ on HMDS treated $n^{+}-\mathrm{Si} / \mathrm{SiO}_{2}$ substrate. The hole transfer curves were derived at drain voltages $\left(\mathrm{V}_{\mathrm{D}}\right)$ of $-70 \mathrm{~V}$.

Table 1. OFET device performance of S10 and S11 thin films annealed at $100{ }^{\circ} \mathrm{C}$, and $120{ }^{\circ} \mathrm{C}$ on HMDS treated $n^{+}-\mathrm{Si}_{/} \mathrm{SiO}_{2}$ substrates using bottom-gate, top-contact (BGTC) device architecture.

\begin{tabular}{ccccc}
\hline \multicolumn{2}{c}{$\begin{array}{c}\text { Annealing } \\
\text { Temperature }\left({ }^{\circ} \mathbf{C}\right)\end{array}$} & $\boldsymbol{\mu}\left(\mathbf{c m}^{2} \cdot \mathbf{V}^{-\mathbf{1}} \cdot \mathbf{s}^{-\mathbf{1}}\right)$ & $\mathbf{V}_{\mathbf{T}}(\mathbf{V})$ & On/Off Ratio \\
\hline \multirow{2}{*}{ S10 } & 100 & $1.0 \times 10^{-5}$ & $-18--25$ & $2.5 \times 10^{3}$ \\
& 120 & $2.2 \times 10^{-4}$ & $-16--20$ & $1.1 \times 10^{4}$ \\
\multirow{2}{*}{ S11 } & 100 & $8.5 \times 10^{-4}$ & $-20--24$ & $4.8 \times 10^{4}$ \\
& 120 & $7.8 \times 10^{-3}$ & $-17--21$ & $1.3 \times 10^{5}$ \\
\hline
\end{tabular}


The surface morphologies of S10 and S11 thin films were studied by atomic force microscopy (AFM) in the tapping mode and are shown in Figure 8. The spin coated films exhibited amorphous domains for both the materials. For S10, upon thermal annealing at $120{ }^{\circ} \mathrm{C}$, the thin films become discontinuous due to strong aggregation, which deteriorates the charge transport properties. Compared to S10, S11 exhibited an interconnected network, which is beneficial for charge carrier transport. Two dimensional X-ray diffraction measurements of these two films were further investigated in order to study the crystallinity and microstructure of these films (see Supplementary Figure S10). It can be seen that both thin films exhibited week diffraction peaks. After thermal annealing, the diffraction intensity was enhanced for S10, which is consistent with the AFM image. However, for S11, the thin film still showed weak diffraction peak after thermal annealing, indicating its amorphous nature. These results are consistent with the results of surface morphologies as well as slight low charge transport properties.
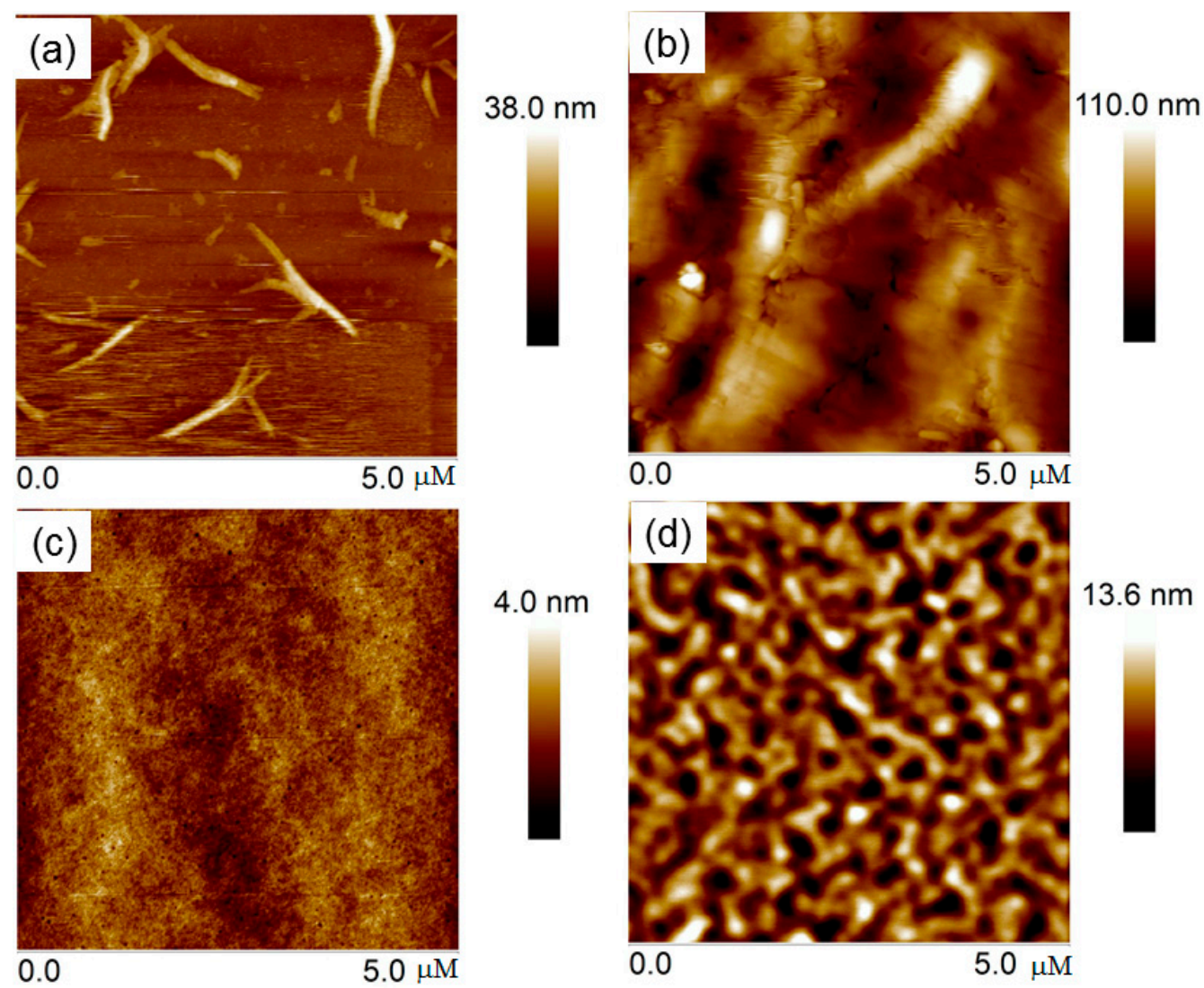

Figure 8. The AFM images of $\mathbf{S 1 0}(\mathbf{a}, \mathbf{b})$ and $\mathbf{S 1 1}(\mathbf{c}, \mathbf{d})$ for as-cast and thermally annealed films.

\section{Experimental Section}

\subsection{Materials and Instruments}

All the reagents and chemicals used, unless otherwise specified, were purchased from Sigma-Aldrich Co. (Sydney, Australia). The solvents used for reactions were obtained from Merck Specialty Chemicals (Sydney, Australia) and were used as such. (E)-6,6'-dibromo-1,1'-bis(2-ethylhexyl)-(3,3'biindolinylidene)-2,2'-dione was purchased from Luminescence Technology Corporation (LTC, Taiwan) and was used as such. Unless otherwise specified, all ${ }^{1} \mathrm{H}$ - and ${ }^{13} \mathrm{C}-\mathrm{NMR}$ spectra were recorded using a Bruker AV400 spectrometer (Bruker Corporation, Billerica MA, USA) at $400 \mathrm{MHz}$ and 
100.6 MHz, respectively. Chemical shifts $(\delta)$ are measured in parts per million (ppm). Thin layer chromatography (TLC) was performed using $0.25 \mathrm{~mm}$ thick plates precoated with Merck Kieselgel 60 F254 silica gel (Merck, Darmstadt, Germany), and visualized using ultraviolet (UV) light (254 nm and $365 \mathrm{~nm}$, Spectronics Corporation, Westbury, NY, USA). Melting points were measured using a Gallenkamp MPD350 digital melting point apparatus (Sanyo, Osaka, Japan) and are uncorrected. High-resolution mass spectra (atmospheric-pressure chemical ionization (APCI)) experiments were performed with a thermo scientific Q Exactive Fourier-transform mass spectrometer (Thermo Scientific, Bremen, Germany), ionizing by APCI from an atmospheric solids analysis probe (ASAP) [33]. UV-Vis absorption spectra were recorded using a Hewlett Packard HP 8453 diode-array UV-Vis spectrometer (Agilent Technologies, Mulgrave Victoria, Australia). Work functions of all the materials were estimated using PESA. PESA measurements were recorded using a Riken Keiki AC-2 PESA spectrometer (RKI Instruments, Union City, CA, USA) with a power setting of $5 \mathrm{nW}$ and a power number of 0.5 . Samples for PESA were prepared on cleaned glass substrates. The thermal stability of S10 and S11 was investigated by TGA and DSC.

\subsection{Cyclic-Voltammetry}

CV was carried out in freshly distilled dichloromethane (over calcium hydride), with a supporting electrolyte of $0.1 \mathrm{M}$ tetrabutylammoniumhexafluorophosphate (Bu4NPF 6 , Electrochemical grade, Aldrich), which was twice recrystallized from ethanol before use. A glassy carbon electrode was used as a working electrode (ALS, Tokyo, Japan), which was polished with $0.05 \mu \mathrm{M}$ alumina on a felt pad, washed with distilled water followed by ethanol and dried under a $\mathrm{N}_{2}$ stream before use. A platinum wire was used as a counter electrode and a silver wire was used as a pseudo reference electrode. Ferrocene was used as an internal reference, by doping all solutions with an approximately equimolar amount of ferrocene. Reported voltammograms were recorded at a scan rate of $50 \mathrm{mV} \cdot \mathrm{s}^{-1}$. Redox potentials ( $\mathrm{E}_{1 / 2}$ values) were taken as a half-way point between forward and reverse peaks for each reversible redox process.

\subsection{Device Preparation for Thin Film Transistors}

Top contact/bottom gate OFET devices fabricated using $n^{+}-\mathrm{Si} / \mathrm{SiO}_{2}$ substrates where $n^{+}-\mathrm{Si}$ and $\mathrm{SiO}_{2}$ work as gate electrode and gate dielectric, respectively. The thickness of thermally grown silicon oxide layer is around $\sim 200 \mathrm{~nm}$ with a capacitance of about $17 \mathrm{nF} / \mathrm{cm}^{2}$. The $\mathrm{SiO}_{2} / \mathrm{Si}$ substrate was cleaned with acetone followed by isopropyl alcohol. It was then immersed in a piranha solution $\left(\mathrm{V}\left(\mathrm{H}_{2} \mathrm{SO}_{4}\right): \mathrm{V}\left(\mathrm{H}_{2} \mathrm{O}_{2}\right)=2: 1\right)$ for $20 \mathrm{~min}$, followed by rinsing with deionized water, and then immersed in a $0.1 \mathrm{M}$ solution of hexamethyldisilazane (HMDS) in anhydrous toluene at $60{ }^{\circ} \mathrm{C}$ for $30 \mathrm{~min}$. It was then rinsed with toluene followed by drying under nitrogen stream. Device fabrication was completed by deposition of $\mathbf{S 1 0}$ and $\mathbf{S 1 1}$ by spin coating $\mathrm{CHCl}_{3}$ solution $(0.5 \mathrm{wt} \%)$ at $3000 \mathrm{rpm}$ for $1 \mathrm{~min}$. Subsequently, on top of the $\mathbf{S 1 0}$ and $\mathbf{S 1 1}$ active layer, a $100 \mathrm{~nm}$ thick gold (Au) thin film was deposited for source (S) and drain (D) electrodes through a shadow mask. For a typical OFET device reported here, the source-drain channel length (L) and channel width (W) was $100 \mu \mathrm{m}$ and $1 \mathrm{~mm}$, respectively. The device characteristics of the OFETs were measured at room temperature under nitrogen with a Keithley 4200 source meter. The field effect mobility $(\mu)$ was calculated from the saturation regime of 
transfer characteristics. Estimation of the carrier mobility was done using the standard transistor Equation (1) in saturation mode:

$$
\mathrm{ID}_{\mathrm{D}}=\mathrm{W} /(2 \mathrm{~L}) \mathrm{C}_{\mathrm{i}} \mu\left(\mathrm{V}_{\mathrm{G}}-\mathrm{V}_{\mathrm{T}}\right)^{2}
$$

where $\mu$ is the field effect mobility, $\mathrm{L}$ is channel length, $\mathrm{W}$ is channel width, $\mathrm{C}_{\mathrm{i}}$ is the gate insulator capacitance.

\subsection{Synthesis and Characterisation of Target Molecules}

Both the materials, S10 and S11, were synthesized by reacting the bis-bromoisoindigo precursor, (E)-6,6'-dibromo-1,1'-bis(2-ethylhexyl)-(3,3'-biindolinylidene)-2,2'-dione, at reflux, with 4-(diphenylamino) phenyl)boronic acid and carbazole in dimethoxyethane and toluene solvents, respectively. The reaction strategy is depicted in Scheme 1. Both the materials were purified through conventional column chromatography on silica and their chemical structures were confirmed via spectroscopic analyses.

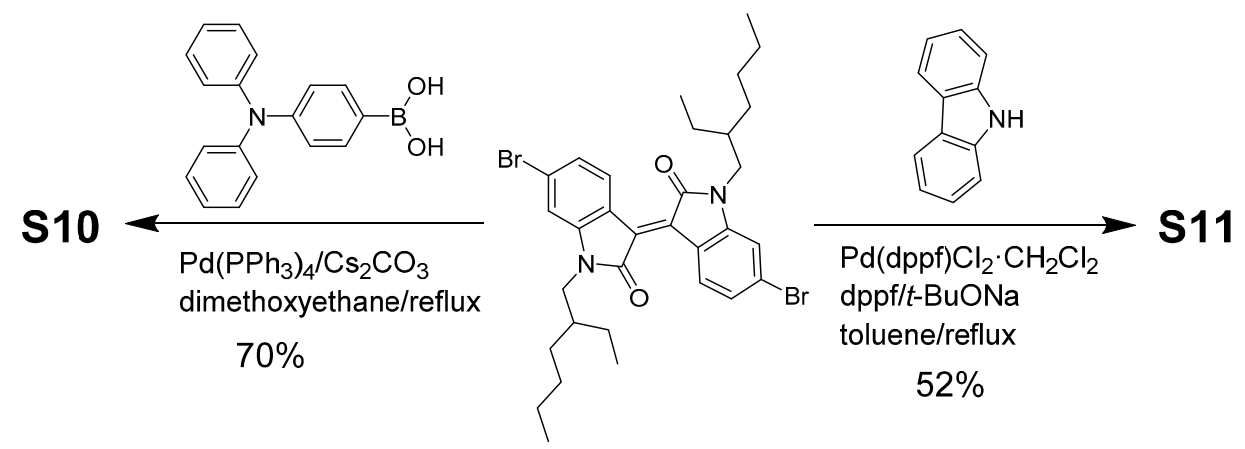

Scheme 1. Reaction scheme for the synthesis of S10 and S11.

(E)-6,6'-bis(4-(Diphenylamino)phenyl)-1-(2-ethylheptyl)-1'-(2-ethylhexyl)-(3,3'-biindolinylidene)-2,2'dione (S10). (E)-6,6'-dibromo-1,1'-bis(2-ethylhexyl)-(3,3'-biindolinylidene)-2,2'-dione (0.25 g, $0.38 \mathrm{mmol})$ and (4-(diphenylamino)phenyl)boronic acid $(0.27 \mathrm{~g}, 0.95 \mathrm{mmol})$ were mixed in dimethoxyethane $(25 \mathrm{~mL})$ in a $100 \mathrm{~mL}$ round-bottomed flask at room temperature and the reaction mixture was stirred for $15 \mathrm{~min}$ followed by the addition of cesium carbonate $\left(\mathrm{Cs}_{2} \mathrm{CO}_{3}\right)(0.37 \mathrm{~g}, 1.14 \mathrm{mmol})$. The resulting suspension was degassed for $10 \mathrm{~min}$ by purging with argon, and tetrakis(triphenylphosphine)palladium $(0)\left(\operatorname{Pd}\left(\mathrm{PPh}_{3}\right)_{4}\right)$ catalyst $(0.10 \mathrm{~g})$ was added to the reaction mixture. The reaction mixture was refluxed in an oil bath for $12 \mathrm{~h}$ in the absence of light and the progress of reaction was followed by thin layer chromatography, which indicated the consumption of starting dibromo derivative. The reaction mixture was cooled to room temperature, diluted with water $(50 \mathrm{~mL})$, and the product was extracted in chloroform. The organic layer was washed with water followed by brine, dried over anhydrous $\mathrm{MgSO}_{4}$, and recovered to get crude solid which was purified by column chromatography on silica (hexane: ethyl acetate 9:1 as eluent) to afford $\mathbf{S 1 0}(0.26 \mathrm{~g}, 70 \%)$ as a bluish-black solid. IR (thin solid film, $\mathrm{cm}^{-1}$ ): 3034, 2957, 2928, 2858, 1693, 1608, 1591, 1518, 1493, 1455, 1357, 1330, 1281, 1179, 1109, 818, 754; ${ }^{1} \mathrm{H}-\mathrm{NMR}$ (400 MHz, $\left.\mathrm{CD}_{2} \mathrm{Cl}_{2}, \mathrm{TMS}\right): \delta(\mathrm{ppm})$ 9.22-9.19 (2H, m), 7.58-7.56 (4H, m), 7.32-7.25 (10H, m), 7.15-7.05 (16H, m), 7.04-7.00 (2H, m), 3.78-3.67 (4H, m), 1.97-1.86 (2H, m), 1.46-1.26 (16H, m), $0.97-0.93(6 \mathrm{H}, \mathrm{m}), 0.90-0.86(6 \mathrm{H}, \mathrm{m}) ;{ }^{13} \mathrm{C}-\mathrm{NMR}$ (400 MHz, $\left.\mathrm{CD}_{2} \mathrm{Cl}_{2}, \mathrm{TMS}\right): \delta$ (ppm) 168.63, 148.13, $147.39,145.87,144.06,133.71,131.93,129.95,129.31,127.56,124.76,123.55,123.07,120.53$, 
119.60, 105.78, 44.00, 37.69, 30.71, 28.72, 24.09, 23.05, 13.79, 10.47; HRMS (APCI): [M] $]^{+}$, found 972.5338. $\mathrm{C}_{68} \mathrm{H}_{68} \mathrm{~N}_{4} \mathrm{O}_{2}$ requires 972.5337; Elemental Analysis for $\mathrm{C}_{68} \mathrm{H}_{68} \mathrm{~N}_{4} \mathrm{O}_{2}$ : Calculated C, 83.91; $\mathrm{H}$, $7.04 ; \mathrm{N}, 5.76 ; \mathrm{O}, 3.29$; Found C, 83.84; H, 7.01; N, 5.71; O, 3.27.

(E)-6,6'-di(9H-Carbazol-9-yl)-1-(2-ethylheptyl)-1'-(2-ethylhexyl)-(3,3'-biindolinylidene)-2,2'-dione (S11). (E)-6,6'-dibromo-1,1'-bis(2-ethylhexyl)-(3,3'-biindolinylidene)-2,2'-dione (0.25 g, $0.38 \mathrm{mmol})$ was added to the mixture of carbazole $(0.15 \mathrm{~g}, 0.87 \mathrm{mmol})$, sodium $t$-butoxide $(0.11 \mathrm{~g}, 1.14 \mathrm{mmol})$, and (1,1'-bis(diphenylphosphino)ferrocene)dichloropalladium(II), complex with dichloromethane $\left(\mathrm{Pd}(\mathrm{dppf}) \mathrm{Cl}_{2} \cdot \mathrm{CH}_{2} \mathrm{Cl}_{2}\right) \quad(0.081 \mathrm{~g}, 0.1 \mathrm{mmol})$ in toluene $(25 \mathrm{~mL})$ followed by the addition of 1,1'-bis(diphenylphosphino)ferrocene $(0.22 \mathrm{~g}, 0.4 \mathrm{mmol})$ at room temperature, and the resulting suspension was refluxed overnight. The reaction mixture was cooled to room temperature, filtered through Celite bed and the solvent was evaporated off to get crude solid, which was subjected to column chromatography on silica (10\% ethyl acetate/hexane) to afford title compound $\mathbf{S 1 1}(0.161 \mathrm{~g}$, 52\%) as a black solid. IR (thin solid film, $\mathrm{cm}^{-1}$ ): 3057, 2958, 2928, 2872, 1694, 1610, 1501, 1447, 1384, 1334, 1224, 1112, 1099, 736; ${ }^{1} \mathrm{H}$ NMR (400 MHz, $\left.\mathrm{CD}_{2} \mathrm{Cl}_{2}, \mathrm{TMS}\right): \delta(\mathrm{ppm}) 9.50-9.48$ (2H, m), 8.18-8.16 (4H, m), 7.63-7.61 (4H, m), 7.49-7.45 (4H, m), 7.35-7.31 (6H, m), 7.10-7.09 (2H, m), 3.80-3.66 (4H, m), 1.97-1.87 (2H, m), 1.48-1.22 (16H, m), 0.96-0.92 (6H, m), 0.86-0.82 (6H, m); ${ }^{13} \mathrm{C}-\mathrm{NMR}\left(400 \mathrm{MHz}, \mathrm{CD}_{2} \mathrm{Cl}_{2}\right.$, TMS): $\delta$ (ppm) 168.50, 146.88, 141.07, 140.21, 132.19, 131.23, 126.17, $123.76,120.48,120.31,120.21,119.28,110.20,106.12,44.38,37.81,30.74,28.74,24.04,23.00$, 13.74, 10.41; HRMS (APCI): [M] $]^{+}$, found 816.4397. $\mathrm{C}_{56} \mathrm{H}_{56} \mathrm{~N}_{4} \mathrm{O}_{2}$ requires 816.4398; Elemental Analysis for $\mathrm{C}_{56} \mathrm{H}_{56} \mathrm{~N}_{4} \mathrm{O}_{2}$ : Calculated $\mathrm{C}, 82.32 ; \mathrm{H}, 6.91 ; \mathrm{N}, 6.86 ; \mathrm{O}, 3.92$; Found $\mathrm{C}, 82.27 ; \mathrm{H}, 6.86 ; \mathrm{N}$, $6.79 ; \mathrm{O}, 3.89$.

\section{Conclusions}

In conclusion, we have demonstrated the use of isoindigo accepting functionality to generate new D-A-D modular small organic molecules, S10 and S11, which contain a common isoindigo core unit and varied donor functionalities. The new materials, S10 and S11, were synthesized, found to be highly soluble in a variety of common organic solvents, and were thermally stable. Use of a TPA unit as an energy antenna helped to achieve a substantial red-shift of the absorption maximum in the visible region, improved the solubility of target material and helped to reduce optical band-gap. Upon testing these materials as active layers in OFET devices, hole mobilities of the order of $2.2 \times 10^{-4} \mathrm{~cm}^{2} / \mathrm{Vs}$ and $7.8 \times 10^{-3} \mathrm{~cm}^{2} / \mathrm{Vs}$ were achieved for $\mathbf{S 1 0}$ and $\mathbf{S 1 1}$, respectively. There are several reports on isoindigobased polymers but not much work has been done so far on the design and development of small molecules. The reported charge-carrier mobility values for S10 and S11 are promising and through further visionary designing, high mobility values can be achieved. Our results on the effect of donor types on electronic properties of D-A-D modular organic materials can indeed inform the design of futuristic materials for organic electronic applications. Future studies will focus on the use of unsymmetrical donor units and incorporation of $\pi$-spacers between donor and acceptor functionalities.

\section{Supplementary Materials}

Supplementary materials can be accessed at: http://www.mdpi.com/1420-3049/20/09/17362/s1. 


\section{Acknowledgments}

S.V.B. (RMIT) acknowledges financial support from the Australian Research Council (ARC), Australia, under a Future Fellowship Scheme (FT110100152). The CSIRO Division of Materials Science and Engineering (now Manufacturing Flagship), Clayton Victoria Australia is acknowledged for providing support through a visiting fellow position (A.G.). P.S. is thankful to the ARC Future Fellowship Scheme (FT130101337) at Queensland University of Technology, Brisbane, Queensland.

\section{Author Contributions}

H.P. synthesized S10 and S11, and fabricated the organic electronic devices, J.C., A.G., J.W. and P.S. measured mobilities of the devices, A.B. performed DFT calculations, and S.V.B. designed, supervised and analysed the whole data. All the authors contributed for manuscript preparation.

\section{Conflicts of Interest}

The authors declare no conflicts of interest.

\section{References}

1. Wu, H.; Ying, L.; Yang, W.; Cao, Y. Progress and perspective of polymer white light-emitting devices and materials. Chem. Soc. Rev. 2009, 38, 3391-3400.

2. Pron, A.; Gawrys, P.; Zagorska, M.; Djurado, D.; Demadrille, R. Electroactive materials for organic electronics: Preparation strategies, structural aspects and characterisation techniques. Chem. Soc. Rev. 2010, 39, 2577-2632.

3. Hadfield, R.H. Single-photon detectors for optical quantum information applications. Nat. Photon. 2009, 3, 696-705.

4. Cheng, Y.J.; Yang, S.H.; Hsu, C.S.; Synthesis of conjugated polymers for organic solar cell applications. Chem. Rev. 2009, 109, 5868-5923.

5. Gupta, A.; Watkins, S.E.; Scully, A.D.; Singh, T.B.; Wilson, G.J.; Rozanski, L.J.; Evans, R.A. Band-gap tuning of pendant polymers for organic light-emitting devices and photovoltaic applications. Synth. Met. 2011, 161, 856-863.

6. Helgesen, M.; Søndergaard, R.; Krebs, F.C. Advanced materials and processes for polymer solar cell devices. J. Mater. Chem. 2012, 20, 36-60.

7. Li, Y.; Guo, Q.; Li, Z.; Pei, J.; Tian, W. Solution processable D-A small molecules for bulk-heterojunction solar cells. Energ. Environ. Sci. 2010, 3, 1427-1436.

8. Mishra, A.; Bäuerle, P. Small molecule organic semiconductors on the move: Promises for future solar energy technology. Angew. Chem. Inter. Ed. 2012, 51, 2020-2067.

9. Rybakiewicz, R.; Djurado, D.; Cybulski, H.; Dobrzynska, E.; Kulszewicz-Bajer, I.; Boudinet, D.; Verilhac, J.-M.; Zagorska, M.; Pron, A. Arylene bisimides with triarylamine $N$-substituents as new solution processable organic semiconductors: Synthesis, spectroscopic, electrochemical and electronic properties. Synth. Met. 2011, 161, 1600-1610.

10. Marks, T.J.; Ratner, M.A. Design, synthesis, and properties of molecule-based assemblies with large second-order optical nonlinearities. Angew. Chem. Int. Ed. 1995, 34, 155-173. 
11. Marder, S.R.; Kippelen, B.; Jen, A.K.Y.; Peyghambarian, N. Design and synthesis of chromophores and polymers for electro-optic and photorefractive applications. Nature 1997, 388, 845-851.

12. Facchetti, A. $\pi$-Conjugated polymers for organic electronics and photovoltaic cell applications. Chem. Mater. 2011, 23, 733-758.

13. Günes, S.; Neugebauer, H.; Sariciftci, N.S. Conjugated polymer-based organic solar cells. Chem. Rev. 2007, 107, 1324-1338.

14. Lin, Y.; Li, Y.; Zhan, X. Small molecule semiconductors for high-efficiency organic photovoltaics. Chem. Soc. Rev. 2012, 41, 4245-4272.

15. Gupta, A.; Ali, A.; Bilic, A.; Gao, M.; Hegedus, K.; Singh, T.B.; Watkins, S.E.; Wilson, G.J.; Bach, U.; Evans, R.A. Absorption enhancement of oligothiophene dyes through the use of a cyanopyridone acceptor group in solution-processed organic solar cells. Chem. Commun. 2012, $48,1889-1891$.

16. Gupta, A.; Ali, A.; Singh, B.; Bilic, A.; Bach, U.; Evans, R.A. Molecular engineering for panchromatic absorbing oligothiophene donor- $\pi$-acceptor organic semiconductors. Tetrahedron 2012, 68, 9440-9447.

17. Gupta, A.; Armel, V.; Xiang, W.; Fanchini, G.; Watkins, S.E.; MacFarlane, D.R.; Bach, U.; Evans, R.A. The effect of direct amine substituted push-pull oligothiophene chromophores on dye-sensitized and bulk heterojunction solar cells performance. Tetrahedron 2013, 69, 3584-3592.

18. Kumar, R.J.; Churches, Q.I.; Subbiah, J.; Gupta, A.; Ali, A.; Evans, R.A.; Holmes, A.B. Enhanced photovoltaic efficiency via light-triggered self-assembly. Chem. Commun. 2013, 49, 6552-6554.

19. Patil, H.; Gupta, A.; Bilic, A.; Jackson, S.L.; Latham, K.; Bhosale, S.V. Donor-acceptor-donor modular small organic molecules based on the naphthalenediimide acceptor unit for solutionprocessable photovoltaic devices. J. Electron. Mater. 2014, 43, 3243-3254.

20. Tamayo, A.B.; Dang, X.-D.; Walker, B.; Seo, J.; Kent, T.; Nguyen, T.-Q. A low band gap, solution processable oligothiophene with a dialkylated diketopyrrolopyrrole chromophore for use in bulk heterojunction solar cells. Appl. Phys. Lett. 2009, 94, 103301:1-103301:3.

21. Walker, B.; Tamayo, A.B.; Dang, X.-D.; Zalar, P.; Seo, J.H.; Garcia, A, Tantiwiwat, M.; Nguyen, T.-Q. Nanoscale phase separation and high photovoltaic efficiency in solution-processed, small-molecule bulk heterojunction solar cells. Adv. Funct. Mater. 2009, 19, 3063-3069.

22. Li, Z.; Dong, Q.; Li, Y.; Xu, B.; Deng, M.; Pei, J.; Zhang, J.; Chen, F.; Wen, S.; Gao, Y.; et al. Design and synthesis of solution processable small molecules towards high photovoltaic performance. J. Mater. Chem. 2011, 21, 2159-2168.

23. Shi, Q.; Cheng, P.; Li, Y.; Zhan, X. A solution processable D-A-D molecule based on thiazolothiazole for high performance organic solar cells. Adv. Energy. Mater. 2012, 2, 63-67.

24. Stalder, R.; Mei, J.; Reynolds, J.R. Isoindigo-based donor-acceptor conjugated polymers. Macromolecules 2010, 43, 8348-8352.

25. Zhang, G.; Fu, Y.; Xie, Z.; Zhang, Q. Synthesis and photovoltaic properties of new low bandgap isoindigo-based conjugated polymers. Macromolecules 2011, 44, 1414-1420.

26. Lei, T.; Cao, Y.; Fan, Y.; Liu, C.-J.; Yuan, S.-C.; Pei, J. High-performance air-stable organic field-effect transistors: Isoindigo-based conjugated polymers. J. Am. Chem. Soc. 2011, 133, 6099-6101. 
27. Stalder, R.; Mei, J.; Graham, K.R.; Estrada, L.A.; Reynolds, J.R. Isoindigo, a versatile electron-deficient unit for high performance organic electronics. Chem. Mater. 2014, 26, 664-678.

28. Deng, P.; Zhang, Q. Recent developments on isoindigo-based conjugated polymers. Polym. Chem. 2014, 5, 3298-3305.

29. Lei, T.; Wang, J.-Y.; Pei, J. Design, synthesis, and structure-property relationships of isoindigo-based conjugated polymers. Acc. Chem. Res. 2014, 47, 1117-1126.

30. Tian, H.; Yang, X.; Cong, J.; Chen, R.; Liu, J.; Hao, Y.; Hagfeldt, A.; Sun, L. Tuning of phenoxazine chromophores for efficient organic dye-sensitized solar cells. Chem. Commun. 2009, 41, 6288-6290.

31. Frisch, M.J.; Trucks, G.W.; Schlegel, H.B.; Scuseria, G.E.; Robb, M.A.; Cheeseman, J.R.; Scalmani, G.; Barone, V.; Mennucci, B.; Petersson, G.A.; et al. Gaussian 09, revision D.01; Gaussian Incorporation: Wallingford, CT, USA, 2013.

32. Dasari, R.R.; Dindar, A.; Lo, C.K.; Wang, C.-Y.; Quinton, C.; Singh, S.; Barlow, S.; Reynolds, J.R.; Fuentes-Hernandez, C.; Kippelen, B.; et al. Tetracyano isoindigo small molecules and their use in n-channel organic field-effect transistors. Phys. Chem. Chem. Phys. 2014, 16, 19345-19350.

33. McEwen, C.N.; McKay, R.G.; Larsen, B.S. Analysis of solids, liquids, and biological tissues using solids probe introduction at atmospheric pressure on commercial LC/MS instruments. Anal. Chem. 2007, 77, 7826-7831.

Sample Availability: Samples of the compounds S10 and S11 are available from the authors.

(C) 2015 by the authors; licensee MDPI, Basel, Switzerland. This article is an open access article distributed under the terms and conditions of the Creative Commons Attribution license (http://creativecommons.org/licenses/by/4.0/). 